\title{
EPIDEMIOLOGIA DA TENÍASE/CISTICERCOSE POR Taenia solium E Taenia saginata
}

\author{
EPIDEMIOLOGY OF TENIASIS/CYSTICERCOSIS \\ BY Taenia solium AND Taenia saginata
}

\author{
Márcia Regina Pfuetzenreiter ${ }^{1}$ Fernando Dias de Ávila-Pires ${ }^{2}$
}

\section{- REVISÃO BIBLIOGRÁFICA -}

\section{RESUMO}

No presente artigo, os autores fazem uma revisão dos aspectos epidemiológicos da teníase e cisticercose. A cisticercose é produzida pelo desenvolvimento da forma larval da Taenia, o Cysticercus, nos tecidos, sendo transmitida pela ingestão de ovos de Taenia. A cisticercose humana e animal são consideradas um grande problema sócio-econômico em muitos países. É considerada uma zoonose endêmica, estando distribuída nos países em desenvolvimento, especialmente nas áreas rurais. A invasão da larva no sistema nervoso central em humanos constitui uma séria complicação. A cisticercose é um dos maiores problemas de saúde pública dos países em desenvolvimento e a neurocisticercose é considerada a doença parasitária mais comum do sistema nervoso humano. A conservação da carne em temperatura inferior $a-15^{\circ} \mathrm{C}$ durante seis dias, sua cocção adequada, além da inspeção sanitária das carnes e o diagnóstico e tratamento da teníase humana em áreas endêmicas constituem as principais medidas de controle.

Palavras-chave: epidemiologia, teníase, cisticercose, neurocisticercose, Taenia solium, Taenia saginata.

\section{SUMMARY}

Is described a review of the epidemiological aspects of teniasis and cysticercosis. Cysticercosis is caused by the development of the larval form of Taenia, wich results in the Cysticercus in tissues, and is transmitted through ingestion of Taenia eggs. Human and animal cysticercosis are a great socioeconomic problem in many countries. It is a endemic

\begin{abstract}
zoonosis and is widespread in developing countries especially in rural areas. Larval invasion of the central nervous system constitutes a serious complication in humans. Cysticercosis is one of the great public health problems in developing countries and the neurocysticercosis is considered the most common parasitic disease of the human central nervous system. The freezing of meat for six days in temperatures below $-15^{\circ} \mathrm{C}$, its adequate cooking, meat inspection and treatment individual patients infected with adult worms in endemic areas are the main control measures.
\end{abstract}

Key words: epidemiology, teniasis, cysticercosis, Taenia solium, neurocysticercosis, Taenia saginata.

\section{INTRODUÇÃO}

Os cestódeos Taenia solium e Taenia saginata são responsáveis pela teníase humana. O gênero Taenia pertence à família Taenidae, à classe Cestoidea e à ordem Cyclophyllidea (REY, 1991). As respectivas formas larvais (Cysticercus cellulosae e Cysticercus bovis - denominação sem valor taxonômico) produzem a cisticercose. O ciclo das tênias implica dois hospedeiros, um definitivo e um intermediário, e uma fase de vida livre. O único hospedeiro definitivo de ambas as tênias (fase adulta do parasito) é o homem, em cujo intestino delgado se alojam. Os hospedeiros intermediários de Taenia

\footnotetext{
${ }^{1}$ Médico Veterinário, Mestre em Saúde Pública, área de concentração Epidemiologia, Centro de Ciências Agroveterinárias, Universidade do Estado de Santa Catarina (UDESC), Av. Luiz de Camões, 2090, 88502-030, Lages, SC, a2mrp@ cav.udesc.br. Autor para correspondência.

${ }^{2}$ Formado em História Natural, Doutor em Ciências, Centro de Ciências da Saúde (CCS), Universidade Federal de Santa Catarina (UFSC).
} 
solium são os suínos e os de $\boldsymbol{T}$. saginata são os bovinos, desenvolvendo-se na musculatura (ACHA \& SZYFRES, 1986; REY, 1991). Há, portanto, três fases com relação à população de parasitas: adulto no hospedeiro definitivo, ovos no ambiente e cisticercos (fase larval) no hospedeiro intermediário (GEMMELL \& LAWSON, 1982; GEMMELL $\boldsymbol{e}$ t al., 1983).

Quando os ovos de tênia são ingeridos pelos hospedeiros intermediários, os embriões (oncosferas) se libertam do ovo no intestino delgado pela ação dos sucos digestivos e bile. As oncosferas penetram na parede intestinal e, em 24 a 72 horas, difundem-se no organismo através da circulação sangüínea. Ocorre então formação de cisticercos nos músculos esqueléticos e cardíaco (GEMMELL $\boldsymbol{e t}$ $\boldsymbol{a l} .$, 1983). Os cistos medem de 7 a $12 \mathrm{~mm}$ de comprimento por 4 a $6 \mathrm{~mm}$ de largura (REY, 1992).

Os parasitas adultos (tênias) são específicos do hospedeiro definitivo, enquanto que as fases larvárias (cisticercos) não são específicos dos hospedeiros intermediários (REY, 1973; REY, 1991). Alguns autores sustentam que a cisticercose humana por cisticercos de $\boldsymbol{T}$. saginata é extremamente rara ou não ocorre, mas não há comprovação científica desse fato (ACHA \& SZIFRES, 1986; BENENSON, 1992; ORGANIZACION PANAMERICANA DE LA SALUD, 1994; SCHANTZ et al., 1994; SCHENONE et al., 1982), enquanto outros admitem a possibilidade de cisticercose humana por ambas as espécies de tênia (GEMMELL et al., 1983; PAWLOWSKI \& SCHULTZ, 1972; REY, 1973; REY, 1991).

\section{TENÍASE HUMANA}

T. solium mede de 3 a 5 metros de comprimento. A cabeça ou escólex é provida de 4 ventosas e rostro armado com dupla coroa de ganchos. Além do escólex, possui o colo ou pescoço (mais delgado) e, finalmente, o estróbilo ou corpo com as proglotes ou anéis. As proglotes se dividem em jovens, maduras e grávidas, estando estas últimas repletas de ovos. As proglotes grávidas medem $1 \mathrm{~cm}$ de comprimento por 0,6 a $0,7 \mathrm{~cm}$ de largura. $\boldsymbol{T}$. saginata mede 6 a 7 metros e não possui ganchos no rostro (CARRADA-BRAVO, 1987; GEMMELL $\boldsymbol{e} t$ al., 1983; HUGGINS, 1989).

A eliminação de proglotes no caso de $\boldsymbol{T}$. solium pode não ser observada, sendo misturada com as fezes, passando despercebida (CARRADABRAVO, 1987; REY, 1991). As proglotes de $\boldsymbol{T}$. saginata são notadas pelo hospedeiro por serem eliminadas ativamente do organismo com movimentos perceptíveis, mostrando ramificações seg- mentares, ao contrário de $\boldsymbol{T}$. solium (HUGGINS, 1989).

As tênias podem viver muitos anos no intestino delgado do homem. No caso de $\boldsymbol{T}$. solium, podem ser eliminadas de três a seis proglotes diariamente. Cada proglote contém uma média de 30.000 a 50.000 ovos. Cada proglote grávida de $\boldsymbol{T}$. saginata contém em torno de 80.000 ovos, sendo que um paciente parasitado pode contaminar o meio ambiente com cerca de 700.000 ovos por dia (REY, 1992).

O homem adquire a tênia ao ingerir carne contaminada crua ou mal cozida contendo cisticercos (GEMMELL et al., 1983). Os cisticercos são liberados durante a digestão da carne e o escólex desenvagina sob ação da bile, fixando-se no intestino delgado. As primeiras proglotes são eliminadas dentro de 60 a 70 dias. A tênia vive no intestino delgado do homem e, normalmente, o hospedeiro alberga apenas um parasita. Isso poderia ser devido à imunidade desenvolvida pelo próprio hospedeiro, impedindo o desenvolvimento de outras tênias da mesma espécie (REY, 1992).

Estão mais sujeitas à teníase as pessoas que preparam alimentos e provam a carne antes de cozinhar e indivíduos que fazem as refeições fora de casa. Fatores econômicos, culturais (hábitos alimentares) e religiosos tendem a expor certos grupos de indivíduos em maior ou menor grau. Na culinária tradicional de muitas culturas, há pratos que utilizam carne crua, por exemplo o quibe cru. (REY, 1991).

As duas espécies de Taenia, segundo ACHA \& SZYFRES (1986) estão distribuídas em todo o mundo. É endêmica na América Latina (GEMMELL et al., 1983). No Brasil, foi detectada uma freqüência média de $1 \%$ entre os anos de 1965 a 1968 e de 3\% entre os anos de 1986 a 1989 (GEMMELL $\boldsymbol{e}$ t $\boldsymbol{a l}$., 1983; ORGANIZACION PANAMERICANA DE LA SALUD, 1994).

A teníase pode se apresentar de forma assintomática, porém alguns pacientes manifestam alterações no apetite (anorexia ou apetite exagerado), náuseas, vômitos, dor abdominal, diarréia, emagrecimento, irritabilidade e fadiga (CARRADABRAVO, 1987; HUGGINS, 1989).

O diagnóstico pode ser realizado através do exame de proglotes nas fezes, pesquisa de ovos nas fezes, ou pesquisa de ovos com a técnica da fita gomada na região perianal. Os ovos das duas espécies de tênia não podem ser diferenciados (HUGGINS, 1989; REY, 1992). As drogas mais utilizadas atualmente para o tratamento são o praziquantel, mebendazol e albendazol (HUGGINS, 1989; REY, 1992). 


\section{CISTICERCOSE EM HOSPEDEIROS NÃO HUMANOS}

Quando os bovinos ou os suínos ingerem os ovos das tênias junto com o pasto ou a água, desenvolvem cisticercos em seus tecidos. $\mathrm{O}$ hábito pouco higiênico das pessoas defecarem diretamente no ambiente, ou em sanitários sem as devidas fossas, muitas delas instaladas sobre córregos e rios, contribui para o problema (ACHA \& SZYFRES, 1986). Para GEMMELL \& LAWSON (1982), a ingestão de ovos pelos animais se dá na maior parte das vezes, por ingestão de fezes. Os bovinos normalmente evitam pastar ao redor de fezes, mas podem, sob condições adversas, por falta de alimentos ingerirem fezes. Já os suínos, por possuírem hábitos coprofágicos, teriam mais facilidade de adquirir a doença. Entretanto, a viabilidade dos ovos no meio ambiente poderia facilitar a infecção sem que, necessariamente, o animal ingira fezes.

As informações sobre cisticercose suína e bovina provêm dos registros da inspeção veterinária de carnes. A inspeção consta de exames de visualização, palpação e cortes dos músculos da cabeça, língua, coração, diafragma, músculos do pescoço e intercostais (BRASIL, 1980). Convém assinalar que a inspeção rotineira dos animais nos frigoríficos tem sérios limites para a identificação de carcaças infectadas, particularmente com infecções leves (GEMMELL et al., 1983; RODRIGUES, 1993; SARTI-GUTIERREZ et $\boldsymbol{a l}$., 1988). A liberação da carcaça ocorre quando da ausência de cisticercos. Quando há presença de cisticercos, ocorre o aproveitamento condicional e até condenação total (BRITO, 1987; GEMMELL et al., 1983; BRASIL, 1980; SECRETARIA DE ESTADO DA AGRICULTURA E ABASTECIMENTO, 1993).

Nas áreas rurais, freqüentemente, os pequenos produtores criam suínos em pequena quantidade, sem controle sanitário e, muitas vezes, com acesso a fezes humanas, o que facilitaria a ingestão de ovos e aquisição da enfermidade (ACEVEDOHERNÁNDEZ, 1982). Contudo, SARTI et al. (1992) não verificaram correlação entre a forma de criação de suínos e a prevalência de cisticercose nestes animais, porque, aparentemente, nem todos os animais criados fechados permanecem durante a vida toda assim, tendo oportunidades de ter contato com fezes humanas. SARTÍ GUTIÉRREZ \& GUTIÉRREZ OSPINA (1986) advertem que os animais criados sem boas condições de higiene, nas pequenas propriedades, em geral são sacrificados pelos próprios donos, sem inspeção veterinária, para o consumo da família ou são vendidos livremente nos mercados.
As perdas econômicas pela cisticercose bovina e suína são consideráveis pela condenação das carcaças contendo cisticercos. UNGAR \& GERMANO (1992) examinaram os dados de fichas dos abatedouros do Estado de São Paulo e encontraram uma prevalência de cisticercose bovina de 5,5\% . Os autores estimam que a prevalência da cisticercose bovina no Brasil está entre 0,7 e 5,3\%.

Nos bovinos, o cisticerco se desenvolve em 60 a 75 dias. Em algumas semanas, ou até 9 meses, os cistos começam a degenerar, morrem e calcificam (ACHA \& SZIFRES, 1986). Nos suínos, o desenvolvimento completo dos cisticercos se dá em 60 dias após a infecção (SALAZARSCHETTINO \& HARO-ARTEAGA, 1990), permanecendo a larva infectante para o homem durante vários anos (REY, 1992).

GEMMELL (1987), utilizando $\boldsymbol{T}$. hydatigena e T. ovis dos ovinos como modelo para o estudo da biologia de $\boldsymbol{T}$. solium e $\boldsymbol{T}$. saginata, concluiu que a resposta imune nos animais depende do tempo entre exposições (ingestão de ovos) e não do número de ovos ingeridos. O conhecimento da imunidade dos animais para a cisticercose não está totalmente desenvolvido, havendo muitos pontos a serem esclarecidos.

Há fatores que auxiliam a dispersão dos ovos tais como: a contaminação fecal do solo, o transporte através do vento, aves, anelídeos e artrópodes (moscas, besouros, traças, formigas, pulgas e ácaros oribatídeos) (LAWSON, 1982; GEMMELL \& LAWSON, 1982; GEMMELL et al., 1983). Os ovos de todas as tênias são sensíveis à dessecação e à temperatura elevada (GEMMELL \& LAWSON, 1982; GEMMELL, 1987), podendo permanecer viáveis na pastagem por períodos de, aproximadamente, 4 até 12 meses (HUGGINS, 1989). Os ovos são resistentes ao tratamento convencional de esgotos (REIFF, 1994; GEMMELL et al., 1983), porém o tratamento convencional da água como floculação, sedimentação e filtração é suficiente para eliminar os ovos (REIFF, 1994). Na utilização de fezes como fertilizantes, a maneira mais prática de inviabilizar os ovos de tênia seria pela elevação da temperatura através da compostação aeróbica, que pode atingir temperaturas de pelo menos $65^{\circ} \mathrm{C}$ por mais de 7 dias (REIFF, 1994). O calor mata os cisticercos, sendo que o C. cellulosae morre a temperaturas de $55^{\circ} \mathrm{C}$, enquanto que o C. bovis morre a $50^{\circ} \mathrm{C}$. Porém, é muito difícil atingir temperaturas muito elevadas no interior de pedaços grossos de carne (REY, 1991). A carne submetida a temperaturas um pouco acima de $0^{\circ} \mathrm{C}$ não afeta a sobrevivência dos cistos de C. cellulosae. Mas, o congelamento por 4 dias a - 
$5^{\circ} \mathrm{C}$ ou 3 dias a $-15^{\circ} \mathrm{C}$, ou ainda um dia a $-24^{\circ} \mathrm{C}$ mata os cisticercos de suínos (SOTELO et al., 1986). O congelamento da carne de suíno ou bovino por mais de 4 dias a temperaturas de $-5^{\circ} \mathrm{C}$ destrói eficazmente os cisticercos (BENENSON, 1992). Porém, REY (1991) admite que os cisticercos morrem em 6 dias quando mantidos a temperatura de $-15^{\circ} \mathrm{C}$, ou inferiores a esta.

A salga também torna os cisticercos inviáveis. No destino de carcaças de bovinos abatidos com cisticercose com número pequeno de cistos, recomenda-se que a carne seja tratada por 21 dias com salmoura, que pode ser reduzida para 10 dias quando for mantida temperatura igual ou inferior a $1^{\circ} \mathrm{C}$ (MINISTÉRIO DA AGRICULTURA, 1980; SECRETARIA DE ESTADO DA AGRICULTURA E ABASTECIMENTO, 1993). A salga destrói os cisticercos contidos na carne de porco em 14 dias quando a mesma é cortada e submersa em salmoura a 25\% (BARTELS, 1971). Entretanto, THORNTON (1969) cita um tempo mais prolongado de 3 a 4 semanas e os pedaços de carne não devem pesar mais que 2,5kg. PESSOA \& MARTINS (1982) admitem que o uso de salmoura $(50 \mathrm{~g}$ de sal por $\mathrm{kg}$ de carne) durante 2 a 3 semanas inviabiliza os cisticercos.

\section{CISTICERCOSE HUMANA}

A importância do complexo teníasecisticercose para a saúde pública resulta de que o homem, além de hospedeiro definitivo da tênia, pode se tornar hospedeiro intermediário e abrigar a fase larval. É o que se denomina de cisticercose humana (ACHA \& SZIFRES, 1986; REY, 1991). Após um a três dias da ingestão de ovos, ocorre liberação dos embriões no duodeno e jejuno. As larvas alcançam a circulação sangüínea e se fixam nos diversos tecidos (REY, 1992).

VERONESI $\boldsymbol{e}$ t al. (1991) enfatizam que a importância da cisticercose na patologia humana está na dependência da localização do parasita em tecidos nobres, como os do globo ocular e do sistema nervoso central (neurocisticercose), sendo que em outras localizações, como a subcutânea, a muscular e a visceral, o cisticerco representa, de regra, achado sem maior significação. Porém, a presença de cistos nessas localizações poderia ser um indicador da presença de cistos nos tecidos mais nobres.

ZENTENO-ALANIS (1982) categoriza os pacientes com cisticercose de acordo com a localização dos parasitas. Há a forma disseminada (com localização nas vísceras, pele e músculos), a oftalmocisticercose (nos olhos e órbita), a neurocisticer- cose (no sistema nervoso central) e, finalmente, a forma mista com mais de uma das localizações acima citadas. A neurocisticercose ainda pode ser classificada topograficamente em espinhal e cerebral. Os cistos se localizam mais freqüentemente no sistema nervoso central (60 a $90 \%$ dos casos) e o parasita vive entre 18 meses e 2 anos, ou até um período maior. Em 78,6\% dos casos de cisticercose, o parasita se encontra no encéfalo (cisticercose cerebral) (SCHENONE et al., 1982). A cisticercose é a enfermidade parasitária que com maior frequiência afeta o sistema nervoso central (ALBUQUERQUE \& GALHARDO, 1995; BRUTTO \& SOTELO, 1988), sendo considerada a mais grave das infecções parasitárias do sistema nervoso humano (COULDWELL \& APUZZO, 1992; FLISSER \& PLANOARTE, 1991; SCHENONE et al., 1982), visto que acomete grande número de pessoas e produz algumas vezes sintomatologia grave (FLISSER, 1991). O período de incubação é em média de 4 a 8 anos, podendo variar de alguns meses a vários anos (AUBRY et al., 1995).

A cisticercose ocorre nos países da América Central e do Sul, na Ásia, África e Austrália (AUBRY et al., 1995). Nos países em desenvolvimento da Ásia, África, assim como na América Latina, a neurocisticercose é considerada endêmica (BRUTTO \& SOTELO, 1987; BRUTTO \& SOTELO, 1988). Ocorre, esporadicamente, nos países industrializados e nos países de religião muçulmana. Estima-se que, anualmente, são infectadas no mundo cerca de 50 milhões de pessoas com 50.000 mortes (AUBRY et al., 1995). Na América Latina, calcula-se que a taxa de prevalência por neurocisticercose é de 100 casos por 100.000 habitantes, atingindo cerca de 350.000 pessoas (SCHENONE $\boldsymbol{e t}$ al., 1982). A enfermidade foi encontrada em 17 países latino-americanos. De 123.826 necropsias realizadas em nove países, foi encontrada uma taxa de $0,43 \%$ de neurocisticercose, sendo as taxas mais elevadas de morbidade as encontradas no Brasil, Chile, Peru, El Salvador, Guatemala e México, tendo maior frequiência nas áreas rurais (ACHA \& SZIFRES, 1986). Estudos de necropsia em hospitais gerais de áreas endêmicas no México sugerem uma prevalência de 3,8\% (BRUTTO e SOTELO, 1988; WOODHOUSE $\boldsymbol{e t}$ al., 1982). Já GEMMELL et al. (1983) citam uma frequiência mais elevada, entre 0,4 e 3,2\% de neurocisticercose em achados de necropsia na América Latina.

A situação da neurocisticercose em hospitais de neurologia e neurocirurgia no Brasil nos anos de 1947-1955 era de 2,9\%, em 1945-1965, era 
de $3,39 \%$ e, em 1969-1988, era de $3,15 \%$ (ORGANIZACION PANAMERICANA DE LA SALUD, 1994). COSTA-CRUZ et al. (1995) mencionam as freqüências de cisticercose no Brasil variando de $0,12 \%$ a $3,6 \%$, sendo a localização mais frequiente o sistema nervoso central. Segundo ALBUQUERQUE \& GALHARDO (1995), a incidência da neurocisticercose tem sido considerada baixa no nordeste brasileiro, sendo freqüente nos estados do sul, sudeste e centro-oeste do país. Isso pode ser devido à falta de diagnóstico, visto que sob o ponto de vista clínico as manifestações são incaracterísticas.

A enfermidade é descrita em muitos Estados do Brasil (ALBUQUERQUE \& GALHARDO, 1995; CHEQUER \& VIEIRA, 1990; TREVISOLBITTENCOURT et al., 1998; VAZ et al., 1990). No Triângulo Mineiro, Minas Gerais, GOBBI et al. (1980) publicaram estatísticas relatando que em 2.306 necropsias foram encontrados $2,4 \%$ de casos de cisticercose e destes $66 \%$ eram neurocisticercose. COSTA-CRUZ et al. (1995) realizaram 3.937 necropsias em Uberlândia, Minas Gerais, e a análise de 2.862 registros com laudos completos e com idade acima de um ano revelou $1,4 \%$ de cisticercose, sendo $89,7 \%$ com comprometimento do sistema nervoso central isolado ou associado a outras formas clínicas da doença. CLEMENTE \& WERNNECK (1990) calculam que a incidência de neurocisticercose no Rio de Janeiro é de cerca de um caso por mês. Em Lagamar, Minas Gerais, SILVA-VERGARA $\boldsymbol{e t}$ al. (1994) encontraram uma prevalência provável de $1,9 \%$. No município de Ribeirão Preto, Estado de São Paulo, TAKAYANAGUI et al. (1996) obtiveram um coeficiente de prevalência de 54 casos/100.000 habitantes através de notificação compulsória, e CHIMELLI et al. (1998) obtiveram uma prevalência de 67 casos/100.000 habitantes através de necropsias. Foi observado aumento do diagnóstico de neurocisticercose logo após a implantação de serviço de tomografia computadorizada (GALHARDO $\boldsymbol{e t} \boldsymbol{a l} .$, 1993; GONÇALVESCOÊLHO \& COÊLHO, 1996). A incidência da enfermidade continua expressivamente alta e sem tendências para decréscimo (SPINA-FRANÇA $\boldsymbol{e t}$ al., 1993). Na América Latina, o período médio de hospitalização pela neurocisticercose varia entre 42 a 46 dias. Pelo menos 50\% dos casos necessita mais do que uma internação hospitalar e mais do que uma intervenção cirúrgica.

A teníase é um fator importante para o aparecimento da cisticercose humana, havendo interrelação estreita entre teníase e cisticercose (ALARCON EGAS et al., 1988; GARCÍA-ALBEA,
1989; SALAZAR SCHETTINO et al., 1990; SARTI-GUTIERREZ $\boldsymbol{e}$ t al., 1988; SCHENONE $\boldsymbol{e}$ t al., 1982; SILVA-VERGARA $\boldsymbol{e t}$ al., 1994). O homem adquire cisticercose através da ingestão de alimentos contaminados (frutas e verduras) com ovos de tênia, através do uso de água de irrigação contaminada com água de esgoto, ou ainda pela utilização de fezes humanas como adubo. Também pode ocorrer a ingestão de ovos através de água contaminada. Uma outra fonte importante de contaminação são os manipuladores de alimento, que contaminam os alimentos através de maus hábitos higiênicos. O próprio portador de teníase, através de maus hábitos de higiene, também pode se autocontaminar (REIFF, 1994).

Há possibilidade de pessoas que residem em áreas urbanas adquirirem teníase ao freqüentarem o meio rural, pela ingestão de produtos de origem animal contaminados com cisticercos e adquirirem cisticercose pelos alimentos preparados em condições higiências inadequadas, contaminados com ovos de tênia. A ingestão de alimentos artesanais favoreceria a infecção (ORGANIZACION PANAMERICA DE LA SALUD, 1994).

Existe possibilidade também de uma autocontaminação interna através de movimentos antiperistálticos ou vômitos em que os ovos do intestino delgado voltam para o estômago e sofrem ação do suco gástrico, liberando as oncosferas para a corrente circulatória. É a chamada auto-infecção interna. Porém, este mecanismo não está comprovado (ACHA \& SZIFRES, 1986; REY, 1991).

A enfermidade mostrou-se mais freqüente em idades mais avançadas e os pacientes do sexo feminino são acometidos em maior proporção (AGAPEJEV, 1996; NARATA et al., 1998; PFUETZENREITER, 1997). A enfermidade está ligada a hábitos alimentares, sendo mais freqüente em pacientes com maior contato com o meio rural (PFUETZENREITER, 1997). De acordo com NARATA $\boldsymbol{e t}$ al. (1998), na neurocisticercose, o achado clínico mais freqüente é a cefaléia (35,5\%), seguida de epilepsia isolada $(20,9 \%)$ ou associada a outros achados neurológicos $(9 \%)$, podendo, muitas vezes, ocorrer de forma assintomática (CHIMELLI et al., 1998).

O tratamento da neurocisticercose pode ser simplesmente sintomático, ou antiparasitário, ou ainda cirúrgico, dependendo do número, tamanho, localização e grau de atividade dos cistos (ORGANIZACION PANAMERICANA DE LA SALUD, 1994; RODRÍGUES-CARBAJAL et al., 1988). O tratamento antiparasitário pode ser feito com albendazol ou praziquantel (COULDWELL \& APUZZO, 1992; ORGANIZACION PANAMERICANA DE LA SALUD, 1994). 


\section{MEDIDAS GERAIS PARA O CONTROLE}

A aplicação de medidas para o controle da teníase/cisticercose depende das características epidemiológicas da enfermidade na região, incluindo condições econômicas, sociais e culturais. A estratégia fundamental consiste em interromper o ciclo evolutivo do parasita, a fim de evitar a infecção nos animais e na população humana (ORGANIZACION PANAMERICANA DE LA SALUD, 1994). Qualquer controle deve reconhecer a multiplicidade de fatores que interagem para a ocorrência da enfermidade, sejam fatores biológicos, ou o impacto sócioecológico na dinâmica de transmissão (GEMMEL, 1987). As estratégias consistem, fundamentalmente, em: melhoramento das condições de saneamento do meio ambiente; tratamento de toda a população; melhoramento da criação de animais (evitar o acesso de animais a fezes humanas); incrementar a inspeção veterinária de produtos cárneos; evitar o abate e comércio de produtos clandestinos; educação em saúde enfatizando a adoção de hábitos de higiene (GEMMEL et al., 1983; ORGANIZACION PANAMERICANA DE LA SALUD, 1994; REIFF, 1994). Para países endêmicos, além das medidas citadas acima, poderiam ser adotadas medidas para o congelamento da carne com o objetivo de diminuir a transmissão da enfermidade (SOTELO et al., 1986).

Com base nas medidas de controle indicadas acima, é sugerida (PFUETZENREITER, 1997) a implementação de programas de educação em saúde por entidades locais para a população congelar a carne a temperaturas inferiores a $-15^{\circ} \mathrm{C}$ pelo menos durante 6 dias. Essas medidas poderiam ser tomadas no frigorífico, antes mesmo da distribuição da carne para o mercado consumidor, diminuindo a transmissão da enfermidade.

\section{REFERÊNCIAS BIBLIOGRÁFICAS}

ACEVEDO-HERNÁNDEZ, A. Economic impact of porcine cysticercosis. In : FLISSER, A., et al. (Eds.). Cysticercosis: present stat of knowledge and perspectives. New York : Academic, 1982. p.63-67.

ACHA, P., SZIFRES, B. Zoonosis y enfermedades transmisibles comunes al hombre y a los animales. 2 ed. Washington : OPS/OMS, 1986. 989p.

AGAPEJEV, S. Epidemiology of neurocysticercosis in Brazil. Rev Inst Med Trop São Paulo, v.38, n.3, p.207-216, 1996.

ALARCON EGAS, F., ESCALANTE, L., SUAREZ, J. et al. Neurocisticercosis: revisión de 65 pacientes. Arch Neurobiol, v.51, n.5, p.252-268, 1988.

ALBUQUERQUE, E.S. de, GALHARDO, I. Neurocisticercose no Estado do Rio Grande do Norte - relato de oito casos. Arq Neuropsiquiatr, v.53, n.3-A, p.464-470, 1995.
AUBRY, P., BEQUET, D., QUEGUINER, P. La cysticercosis: une maladie parasitaire fréquente et redoutable. Med Trop, v.55, n.1, p.79-87, 1995

BARTELS, H. Inspección veterinaria de la carne. Zaragoza: Acribia, 1971. 491p.

BENENSON, A.S. (Ed.) El control de las enfermedades transmisibles en el hombre. 15 ed. Washington : OPS/OMS, 1992. 618p.

BRASIL. MINISTÉRIO DA AGRICULTURA. Regulamento da inspeção industrial e sanitária de produtos de origem animal. (Aprovado pelo Decreto $\mathrm{n}^{\mathrm{o}}$ 30.691, de 29-3-52, alterado pelo Decreto $\mathrm{n}^{\mathrm{o}}$ 1.255, de 25-6-62). Brasília, 1980 . $174 \mathrm{p}$.

BRITO, D.B. (Coord.). Aspectos clínicos, laboratoriais, epidemiológicos e de controle das teníases/cisticercoses. In: CONGRESSO BRASILEIRO DE ZOONOSES, 1987, Rio de Janeiro, RJ. Anais... Rio de Janeiro : Ministério da Saúde, Associação Brasileira de Especialistas em Zoonoses, 1987. 129p. p.56-60.

BRutTo, O. del, SOTElO, J. Neurocisticercosis. Med Hoy, v.6, n.2, p.21-40, 1987.

BRUTTO, O.H. del, SOTELO, J. Neurocysticercosis : an update. Rev Infect Dis, v.10, n.6, p.1075-1087, 1988.

CARRADA-BRAVO, T. Teniasis-cisticercosis como problema de salud pública. Bol Med Hosp Infant Mex, v.44, n.7, p.427-434, 1987.

CHEQUER, R.S., VIEIRA, V.L.F. Neurocisticercose no Estado do Espírito Santo. Arq Neuro-Psiquiat, v.48, n.4, p.431-440, 1990 .

CHIMELLI, L., LOVALHO, A.F., TAKAYANAGUI, O.M. Neurocysticercosis: contribution of the autopsies in consolidation of the compulsory notification in Ribeirão Preto - SP, Brazil. Arq Neuropsiquiatr, v.56, n.3B, p.577-584, 1998.

CLEMENTE, H.A.M., WERNNECK, A.L. dos S. Neurocisticercose - incidência no Estado do Rio de Janeiro. Arq Neuro-Psiquiat, v.48, n.2, p.207-209, 1990

COSTA-CRUZ, J.M., ROCHA, A., SILVA, A.M. da et al. Ocorrência de cisticercose em necropsias realizadas em Uberlândia, Minas Gerais, Brasil. Arq Neuropsiquiatr, v.53, n.2, p.227-232, 1995.

COULDWELL, W.T., APUZZO, M.L.J. Cysticercosis cerebri. Neurosurg Clin North Am, v.3, n.2, p.471-481, 1992.

FLISSER, A. Teniasis-cysticercosis : an introduction. Southeast Asian J Trop Med Public Health, v.22 (supl.), p.233-235, 1991.

FLISSER, A., PLANOARTE, A. Diagnostico, tratamiento y mecanismos de evasion inmune de la cisticercosis por larvas de Taenia solium en seres humanos y cerdos. Rev Asoc Guatemalteca Parasitol Med Trop, v.6, n.1, p.43-54, 1991.

GALHARDO, I., COUTINHO, M.O. de M., ALBUQUERQUE, E.S. de $\boldsymbol{e t} \boldsymbol{a l}$. A neurocisticercose no Rio Grande do Norte antes e depois da tomografia computadorizada. Arq Neuropsiquiatr, v.51, n.4, p.541-545, 1993.

GARCÍA-ALBEA, E. Cisticercosis en España. Algunos datos epidemiológicos. Rev Clin Esp, v.184, n.1, p.3-6, 1989. 
GEMMELL, M.A., LAWSON, J.R. Ovine cysticercosis: an epidemiological model for the cysticercosis. I. Free-living egg fase. In: FLISSER, A. et al. Cysticercosis: present state of knowledge and perspectives. New York : Academic, 1982. p.87-98.

GEMMELL, M., MATYAS, Z., PAWLOWSKI, Z. et al. (Ed.). Guidelines for surveillance prevention and control of taeniasis/ cysticercosis. Geneva : World Health Organization, 1983. 207p.

GEMMELL, M.A. A critical appoach to the concepts of control and eradication of echinococcosis/hydatidosis and teniasis/cysticercosis. Intern J Parasitol, v.17, n.2, p.465472, 1987.

GOBBI, H., ADAD, S.J., NEVES, R.R. et al. Ocorrência de cisticercose (Cysticercus cellulosae) em pacientes necropsiados em Uberaba, MG. Rev Pat Trop, v.9, n.1-2, p.51-59, 1980.

GONÇALVES-COÊLHO, T.D., COÊLHO, M.D.G. Cerebral cysticercosis in Campina Grande, Paraíba - northern Brazil. Computerized tomography diagnosis importance. Arq Neuropsiquiatr, v.54, n.1, p.94-97, 1996.

HUGGins, D. Teníases. Pediatr Moderna, v.24, n.6, p.251256, 1989.

LAWSON, R. Dispersal of taeniid eggs by blowflies. New Zealand J Zool, v.9, n.1, p.46-47, 1982.

NARATA, A.P., ARRUDA, W.O., UEMURA, E. $\boldsymbol{e} \boldsymbol{t} \boldsymbol{a}$ l. Diagnóstico tomográfico em pacientes neurológicos. Arq Neuropsiquiatr, v.56, n.2, p.245-249, 1998.

ORGANIZACION PANAMERICANA DE LA SALUD. Epidemiologia y control de la teniasis/cisticercosis en America Latina. Washington : OPS/OMS, 1994. 297p.

PAWLOWSKI, Z., SCHULTZ, M. G. Taeniasis and cysticercosis (Taenia saginata). Adv Parasitol, v.10, p.269343, 1972.

PESSÔA, S.B., MARTINS, A.V. Parasitologia médica. 11.ed. Rio de Janeiro: Guanabara Koogan, 1982. 872p.

PFUETZENREITER, M.R. Aspectos sócio-culturais e econômicos de pacientes com diagnóstico preliminar de cisticercose cerebral em Lages, Santa Catarina, Brasil. Florianópolis - SC, 1997. 131p. Dissertação (Mestrado em Saúde Pública) - Centro de Ciências da Saúde, Universidade Federal de Santa Catarina, 1997.

REIFF, F.M. Importance of environmental health measures in the prevention and control of taeniasis and cysticercosis. In : ENCONTRO DO CONE SUL E SEMINÁRIO LATINOAMERICANO SOBRE TENÍASE E CISTICERCOSE, 1994, Curitiba. Anais... Curitiba : Secretaria da Saúde do Paraná, 1994. 191p. p.76-90..

REY, L. Parasitologia. Rio de Janeiro : Guanabara Koogan, 1973. 695p.

REY, L. Parasitologia - parasitos e doenças parasitárias do homem nas Américas e na África. 2 ed. Rio de Janeiro : Guanabara Koogan, 1991. 731p.

REY, L. As bases da parasitologia médica. Rio de Janeiro: Guanabara Koogan, 1992. 349p.
RODRIGUES, L.V.C. Inspeção sanitária e critério de julgamento da cisticercose bovina calcificada. Infecção leve. Ciência Rural, v.23, n.3, p.339-344, 1993.

RODRÍGUES-CARBAJAL, J. et al. La cisticercosis humana en México. Gac Med Mex, v.124, n.5-6, p.191-208, 1988.

SALAZAR-SCHETTINO, P.M., HARO-ARTEAGA, I. Biologia del binomio teniasis-cisticercosis. Bol Chil Parasitol, v.45, n. 3 e 4, p.73-76, 1990.

SALAZAR SCHETTINO, B. et al. Neurocisticercosis y medicina ocupacional. Bol Chil Parasitol, v.45, n.1 e 2, p.812,1990 .

SARTÍ GUTIÉRREZ, E.J., GUTIÉRREZ OSPINA, I. La teniasis y cisticercosis en México (revisión bibliográfica). Salud Publica Mex, v.28, n.5, p.556-563, 1986.

SARTI-GUTIERREZ, E.J. et al. Taenia solium teniasis and cysticercosis in a Mexican village. Trop Med Parasit, v.39, p.194-198, 1988.

SARTI E. et al. Prevalence and risk factors for Taenia solium taeniasis and cysticercosis in humans and pigs in a village in Morelos, Mexico. Am J Trop Med Hyg, v.46, n.6, p.677$685,1992$.

SCHANTZ, P.M., CRUZ, M., SARTI, E. et al. La erradicabilidad potencial de la teniasis y la cisticercosis. Bol Oficina Sanit Panam, v.116, n.5, p.465-469, 1994.

SCHENONE, H., VIllarRoEL, F., ROJAS, A. et al. Epidemiology of human cysticercosis in Latin America. In : FLISSER, A. et al. (Ed.) Cysticercosis: present state of knowledge and perspectives. New York : Academic, 1982. p.25-38.

SECRETARIA DE ESTADO DA AGRICULTURA E ABASTECIMENTO. Regulamentação da inspeção industrial e sanitária de produtos de origem animal. Florianópolis : Secretaria de Estado da Agricultura e Abastecimento, 1993. $174 \mathrm{p}$.

SILVA-VERGARA, M.L., VIEIRA, C. de O., CASTRO, J.H. $\boldsymbol{e}$ al. Achados neurológicos e laboratoriais em população de área endêmica para teníase-cisticercose, Lagamar, MG, Brasil (1992-1993). Rev Inst Med trop São Paulo, v.36, n.4, p.335$342,1994$.

SOTELO, J., ROSAS, N., PALENCIA, G. Freezing of infested pork muscle kills cysticerci. J Am Med Assoc, v.256, n.7, p.893-894, 1986.

SPINA-FRANÇA, A., LIVRAMENTO, J.A., MACHADO, L.R. Cysticercosis of the central nervous system and cerebrospinal fluis. Immunodiagnosis of 1573 patients in 63 years (19291992). Arq Neuropsiquiatr, v.51, n.1, p.16-20, 1993.

TAKAYANAGUI, O.M., CASTRO E SILVA A.A.M.C., SANTIAGO, R.C. et al. Notificação compulsória da cisticercose em Ribeirão Preto - SP. Arq Neuropsiquiatr, v.54, n.4, p.557-564, 1996.

THORNTON, H. Compêndio de inspeção de carnes. Rio de Janeiro : FARMOP, 1969. 665p.

TREVISOL-BITTENCOURT, P.C., SILVA, N.C. da, FIGUEIREDO, R. Prevalence of neurocysticercosis among epileptic in-patients in the west of Santa Catarina - southern Brazil. Arq Neuropsiquiatr, v.56, n.1, p.53-58, 1998. 
UNGAR, M.L., GERMANO, P.M.L. Prevalência da cisticercose bovina no Estado de São Paulo (Brasil). Rev Saúde Públ, v.26, n.3, p.167-172, 1992.

VAZ, A.J., HANASHIRO, A.S.G., CHIEFFI, P.P. $\boldsymbol{e} t \boldsymbol{a l}$. Freqüência de indivíduos com anticorpos séricos antiCysticercus cellulosae em cinco municípios do Estado de São Paulo. Rev Soc Brasil Med Trop, v.23, n.2, p.97-99, 1990.

VERONESI, R., SPINA FRANÇA NETTO, A., FOCACCIA, R. Cisticercose. In: VERONESI, Ricardo. Doenças infecciosas e parasitárias. 8 ed. Rio de Janeiro : Guanabara Koogan,
1991. 1082p.

WOODHOUSE, E., FLISSER, A., LARRALDE, C. Soroepidemiology of human cisticercosis in Mexico. In: FLISSER, A. et al. (Ed.). Cysticercosis: present state of knowlege and perspectives. New York : Academic, 1982, p.11-23.

ZENTENO-ALANIS, G.H. A classification of human cysticercosis. In: FLISSER, A. et al. (Ed.). Cysticercosis: present state of knowledge and perspectives. New York: Academic, 1982. p.107-126

Ciência Rural, v. 30, n. 3, 2000. 